\title{
Effects of curcumin-/boron-basedcompound complexation on antioxidant and antiproliferation activity
}

\author{
Jun Yeong Kim ${ }^{1}$ - Yoon Young Kang ${ }^{1}$ Eun Ji Kim ${ }^{1}$. \\ Joong-Hoon Ahn' ${ }^{1} \cdot$ Hyejung Mok ${ }^{1}$
}

Received: 1 March 2018/Accepted: 10 May 2018/Published online: 26 May 2018

(C) The Korean Society for Applied Biological Chemistry 2018

\begin{abstract}
Simple and reproducible formulation strategies are needed to improve the bio-availability of curcumin. In this study, curcumin was successfully complexed with two boron-based compounds: 2-aminoethyl diphenyl borate (DPBA) and bortezomib (BTZ; Velcade ${ }^{\circledR}$ ). In reversephase high-performance liquid chromatography, DPBA/ curcumin complexes (DPBA/cur) showed delayed elution times compared to those of free curcumin. The UV-visible absorbance peak of DPBA/cur and BTZ and curcumin complexes (BTZ/cur) appeared redshifted. DPBA complexation has a negligible effect on the antioxidant and antiproliferation properties of curcumin for two types of cancer cells: MCF-7 and A549. Thus, curcumin complexation with boron-based compounds could be a method to enhance in vivo stability without loss of bioactivity (i.e., antioxidant and antiproliferation effects).
\end{abstract}

Keywords 2-aminoethyl diphenyl borate - Antioxidant . Antiproliferation · Boron-based compounds · Bortezomib · Curcumin

\section{Introduction}

Curcumin, chemically known as diferuloyl methane, is an active polyphenol mainly derived from turmeric (Curcuma longa) [1]. Curcumin is a well-known antioxidant, antiinflammatory, antimicrobial, and anticarcinogenic, acting by modulating multiple signaling pathways [2]. Despite its

Hyejung Mok

hjmok@konkuk.ac.kr

1 Department of Bioscience and Biotechnology, Konkuk University, Seoul 05029, Republic of Korea great biological activity and safety, curcumin applications have been limited by its poor aqueous solubility (approximately $0.6 \mu \mathrm{g} / \mathrm{ml}$ ) and high vulnerability to autoxidation and physiological metabolism [3]. To address these issues, there have been many studies (e.g., chemical modification of functional groups in curcumin, formulation of curcumin within nanobiomaterials_such as liposomes and nanoparticles-and synthesis of curcumin conjugates) [4-7]. However, simple and reproducible formulation strategies for curcumin are still needed.

Boron has a long history with curcumin. Previously, turmeric extracts or curcumin solutions have been used as colorimetric detecting reagents to determine the amount of boron in water, soil, or foods $[8,9]$. Boron and its compounds (especially boronic acid) form complexes with the keto-enol moiety of curcumin, which can be measured via change in absorbance [10,11]. Despite the wide range of applications for boron-based compounds in several chemical fields, their applications in the biological and pharmacological fields seem few, likely due to the toxicity of boron compounds. However, recent studies reported the safety of several boron-based compounds, which has widened the utility of boron in the biological area [12]. Moreover, pharmaceutical interest in boron has increased for the development of antifungal, antibacterial, and anticancer drugs [13]. Recently, we reported curcumin complexation with 2-aminoethyl diphenyl borate to improve the in vivo and in vitro stability of curcumin [14]. However, comparative evaluation of free curcumin and formulated curcumin in terms of biological activity and biocompatibility should be performed to better understand and broaden the use of curcumin and boron complexes.

In this study, as representative boron-based compounds, 2-aminoethyl diphenyl borate (DPBA) and bortezomib 
(BTZ) were complexed with curcumin at various weight ratios and analyzed by ultraviolet-visible (UV-Vis) spectra. DPBA and curcumin complexes (DPBA/cur) were analyzed using reverse-phase high-performance liquid chromatography (HPLC). To determine the effect of boronic complexation with curcumin on radical scavenging activity, free curcumin and DPBA/cur were analyzed using a 1,1-diphenyl-2-picryl-hydrazyl (DPPH) assay at different curcumin concentrations. The antiproliferation activity of curcumin and DPBA/cur was examined for two types of cells, MCF-7 and A549, to assess the biological activity of curcumin-/boron-based compound complexes.

\section{Materials and methods}

\section{Materials}

Curcumin, dimethyl sulfoxide (DMSO), DPBA, and methanol were purchased from Sigma-Aldrich (St. Louis, MO, USA). Phosphate buffered saline solution was acquired from Gibco BRL (Grand Island, NY, USA). Cell counting kit-8 (CCK-8) reagent was obtained from Dojindo Laboratories (Kumamoto, Japan). DPPH was purchased from Cayman Chemical Company (Ann Arbor, MI, USA). BTZ (Velcade ${ }^{\circledR}$ ) was purchased from Selleck Chemistry (Huston, TX, USA). All other chemicals were of analytical grade.

\section{Reverse-phase HPLC}

Curcumin and DPBA were freshly prepared for every experiment by dissolving them with DMSO. To produce the complexes, curcumin $(50 \mathrm{mg})$ in DMSO was incubated with DPBA at various DPBA/cur weight ratios $(0,1$, and 10) for $5 \mathrm{~min}$. Each sample was diluted to a curcumin concentration of $100 \mu \mathrm{g} / \mathrm{ml}$ in $3 \mu \mathrm{l}$ and analyzed using a HPLC system (Thermo Fisher Scientific, MA, USA) equipped with a photodiode array detector, on a Varian polar C18 reversed-phase column $(4.60 \times 250 \mathrm{~mm}$, $0.45 \mu \mathrm{m}$; Varian, CA, USA). Analytical scale chromatography was used with a mobile phase consisting of $\mathrm{H}_{2} \mathrm{O}$ with $0.1 \%$ formic acid ( $\mathrm{pH} 3.0$ ) and acetonitrile. The percent of acetonitrile in the mobile phase was as follows: $20 \%$ at $0 \mathrm{~min}, 45 \%$ at $10 \mathrm{~min}, 80 \%$ at $20 \mathrm{~min}, 100 \%$ at $20.1 \mathrm{~min}$, $100 \%$ at $25 \mathrm{~min}, 20 \%$ at $25.1 \mathrm{~min}$, and $20 \%$ at $30 \mathrm{~min}$. The flow rate was $1 \mathrm{ml} / \mathrm{min}$. The eluent was detected at a wavelength of 420 and $500 \mathrm{~nm}$, respectively.
UV-Vis absorption spectra of curcumin and boron complexes

The DPBA/cur fraction eluted from the HPLC column was dissolved in DMSO. The absorption spectra were measured on a UV-Vis spectrophotometer (Molecular Devices, Sunnyvale, CA, USA) at wavelengths between 200 and $700 \mathrm{~nm}$ at room temperature. The absorption spectra of free curcumin and DPBA in DMSO were also measured. For BTZ/cur, curcumin (10 mg) was mixed with BTZ at two BTZ/cur weight ratios $(0,1$ and 5$)$ and stirred in methanol for $2 \mathrm{~h}$. After dilution of the reactant with methanol containing $2.5 \mathrm{~N} \mathrm{HCl}$ solution, absorption spectra of curcumin $(10 \mu \mathrm{g} / \mathrm{ml})$ were measured at wavelengths between 200 and $700 \mathrm{~nm}$ at room temperature using a UVVis spectrophotometer. Free curcumin and free BTZ were also measured on a UV-Vis spectrophotometer at wavelengths between 200 and $700 \mathrm{~nm}$ at room temperature according to the processes described above.

\section{DPPH assay}

The percent of free radical inhibition by each substance was assessed using a DPPH assay, based on a previous study [14]. Ascorbic acid (25 mg) for a positive control was dissolved in distilled water (DW) and diluted with methanol to various concentrations $(0,0.4,0.8,1.6,3.1$, 6.2 , and $15.6 \mathrm{mg} / \mathrm{ml})$. For DPBA/cur complexation, curcumin $(50 \mathrm{mg}$ ) was mixed with DPBA (4 mg) and incubated for $5 \mathrm{~min}$. Free curcumin and DPBA/cur in DMSO were diluted with methanol to various concentrations of curcumin $(0,0.05,0.1,0.2,0.4,0.8$, and $2.0 \mathrm{mg} / \mathrm{ml})$. Ascorbic acid, free curcumin, and DPBA/cur were mixed with DPPH solution $(0.04 \mathrm{mg})$ and incubated. The absorbance of each solution was measured at $517 \mathrm{~nm}$ using a plate reader (SpectraMAX, Molecular Devices, CA, USA) to detect changes in color of the DPPH solution.

\section{Antiproliferation activity}

MCF-7 (human breast adenocarcinoma cell line) and A549 (human lung carcinoma cell line) were kept in RPMI 1640 supplemented with $10 \% \mathrm{FBS}, 100 \mathrm{U} / \mathrm{ml}$ penicillin and $100 \mu \mathrm{g} / \mathrm{ml}$ streptomycin at $37{ }^{\circ} \mathrm{C}$ in a humidified atmosphere of $5 \% \mathrm{CO}_{2}$. Both cell lines were seeded at a density of $1 \times 10^{4}$ cells per well in a 96-well plate and incubated for $24 \mathrm{~h}$ at $37{ }^{\circ} \mathrm{C}$. Curcumin $(50 \mathrm{mg})$ and DPBA (4 mg) were mixed at weight ratio of 1:0.08 in DMSO and incubated for $5 \mathrm{~min}$ at room temperature. Free curcumin, DPBA/cur, and a corresponding concentration of free DPBA were used to treat cells at various curcumin concentrations $(0,1,10,25,50,75$, and $100 \mu \mathrm{M})$ in a $10 \%$ serum containing media for $24 \mathrm{~h}$. The control was treated 
with $1 \%$ DMSO in the same media (w/w). After $24 \mathrm{~h}$ of incubation, cell viability was measured using the CCK-8 assay according to the manufacturer's protocol.

\section{Results and discussion}

\section{Preparation of curcumin-/boron-based compound complexes}

To study the bioactivity effect of curcumin complexation with boron-based compounds on curcumin, we selected two boron-based compounds, DPBA and BTZ, as shown in Fig. $1[14,15]$. In our previous study, DPBA was used as a stabilizer for keto-enol tautomer of curcumin, which increased in vivo stability of curcumin [14]. BTZ is a dipeptide boronic acid proteasome inhibitor, which has been used for the treatment of refractory non-small cell lung carcinoma and multiple myeloma [16, 17]. Interestingly, recent studies reported that co-administration of BTZ with curcumin has synergistic therapeutic effects for the treatment of multiple myeloma [18, 19]. However, the molecular mechanism of co-administration of BTZ with curcumin is unknown. Sandra et al. mentioned boronic acid interacts with curcumin during co-treatment with curcumin and bortezomib for cancer therapy [15]. BTZ/cur was analyzed using a UV-Vis spectrometer to examine the interaction of curcumin with boronic acid of BTZ. DPBA/ cur was also characterized using HPLC and a UV-Vis spectrometer. After complexation, bioactivity of curcumin-/boron-based compound complexes was determined using a biochemical DPPH assay and CCK-8 cell viability assay.

\section{Reverse-phase HPLC}

To examine the formation of DPBA/cur, curcumin was mixed with DPBA at different DPBA/cur weight ratios $(0$, 1, and 10) and analyzed by reverse-phase HPLC. Figure $2 \mathrm{~A}$ shows the free curcumin peak at $18.2 \mathrm{~min}$. at a wavelength of $420 \mathrm{~nm}$. As DPBA/cur weight ratio increased, the peak area value of free curcumin decreased accordingly. This result might be attributed to the formation of DPBA/cur complexes. The peak areas of free curcumin were 82.8, 74.8, and 66.3 at a DPBA/cur weight ratio of 0,1 , and 10 , respectively. Considering the absorbance peak of DPBA/cur shifted from 420 to $500 \mathrm{~nm}$ in our previous study, DPBA/cur was also analyzed at a wavelength of $500 \mathrm{~nm}$ [14]. As shown in Fig. 2B, a novel and evident peak was observed in DPBA/cur. The peak area of $\mathrm{DPBA} /$ cur at a DPBA/cur weight ratio of 10 was much larger than that of a DPBA/cur weight ratio of 1 . It is conceivable that DPBA/cur complexes have higher hydrophobicity than free curcumin, which mediated delayed migration of samples in the reverse-phase $\mathrm{C}-18$ column during HPLC. Taken together, we successfully confirmed the formation of DPBA/cur complexes using HPLC.

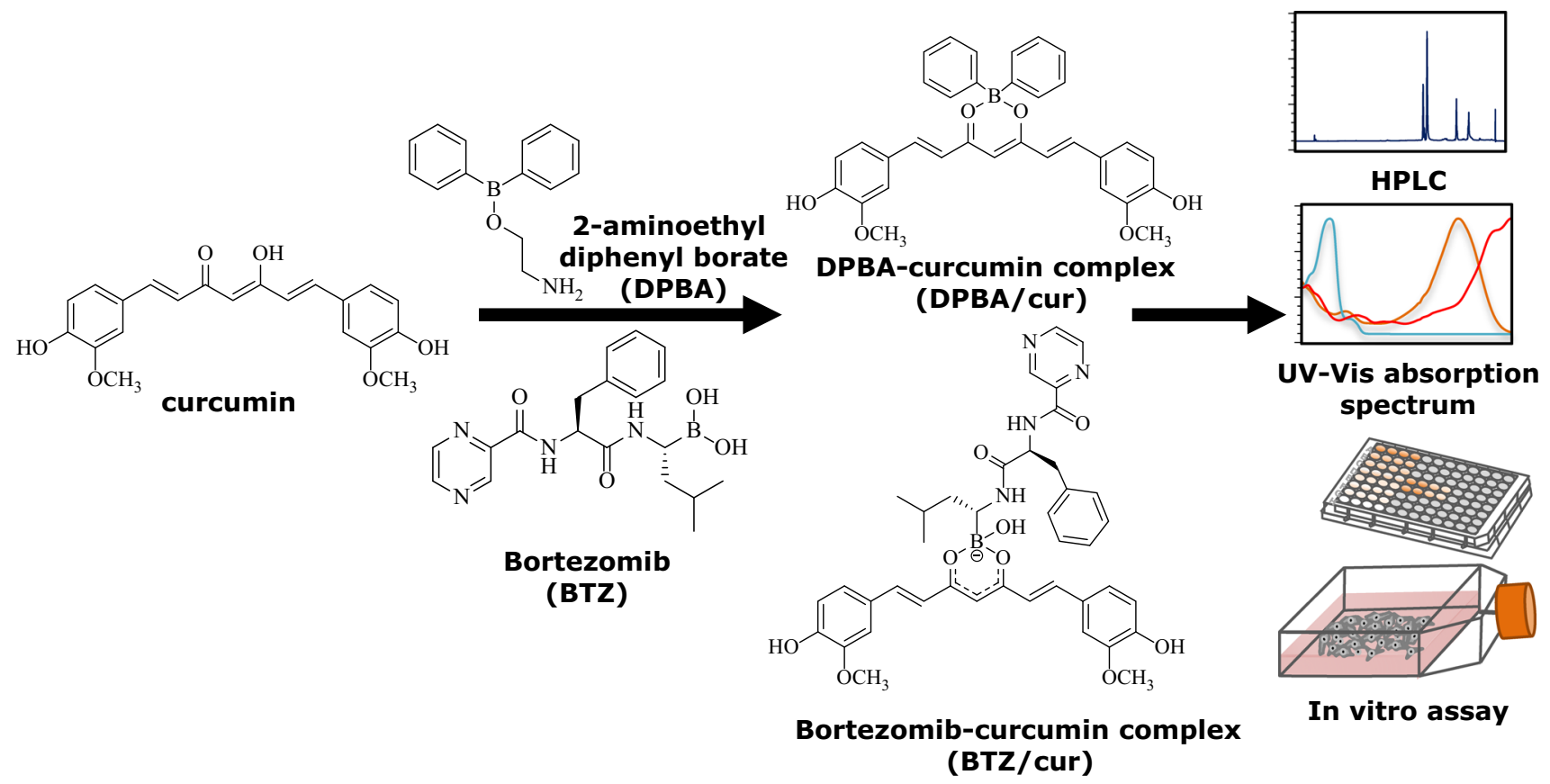

Fig. 1 Schematic illustration of DPBA/cur and BTZ/cur to determine effects on biological activities of curcumin 

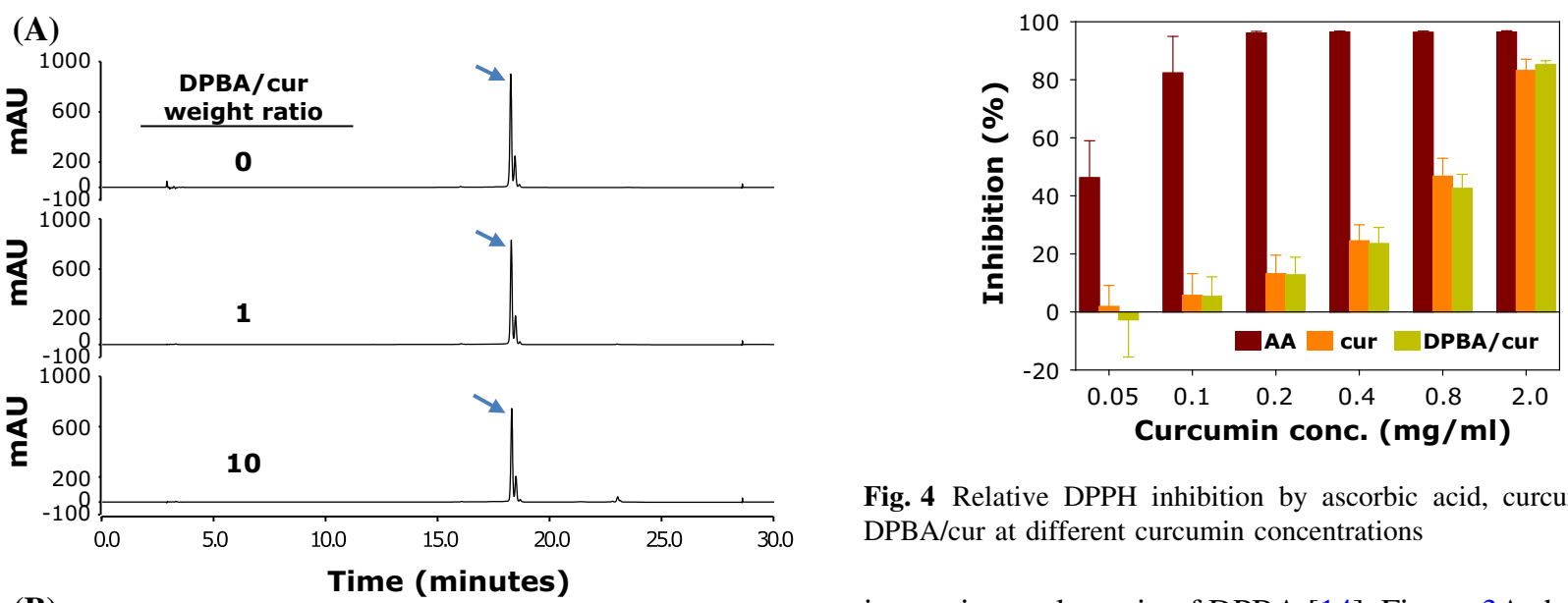

(B)
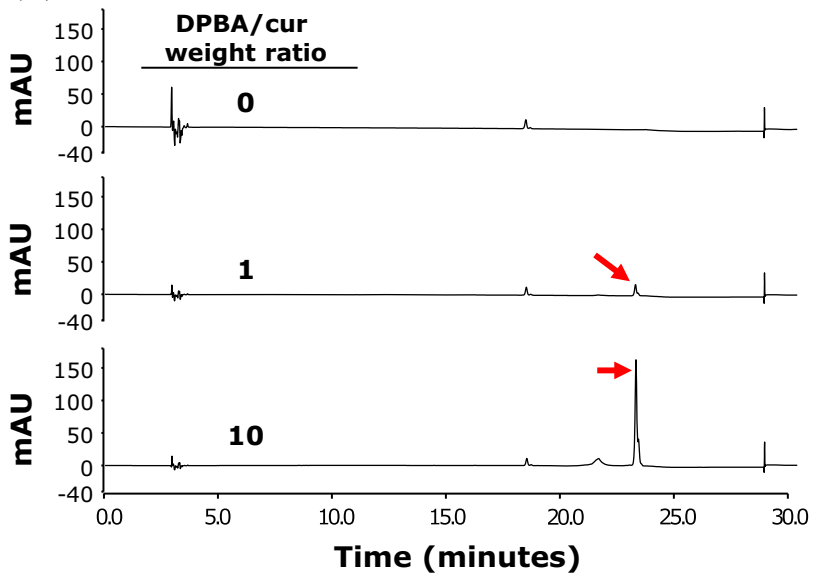

Fig. 2 HPLC analysis of DPBA/cur samples at different DPBA/cur weight ratios at a wavelength of (A) $420 \mathrm{~nm}$ and (B) $500 \mathrm{~nm}$

\section{UV-Vis absorption spectra of curcumin complexes}

After HPLC analysis, the fraction eluted from the HPLC column was dissolved in DMSO to measure UV-Vis absorption spectra. According to previous studies, curcumin has the tendency to redshift after interaction with boron-based compounds [20, 21]. In our previous study, gradual redshift of curcumin was observed with the

Fig. 4 Relative DPPH inhibition by ascorbic acid, curcumin, and DPBA/cur at different curcumin concentrations

increasing molar ratio of DPBA [14]. Figure 3A shows that DPBA/cur exhibited redshifted absorption spectra—having its highest peak at a wavelength of $514 \mathrm{~nm}$. As expected, free DPBA showed no noticeable absorbance at a wavelength of $500 \mathrm{~nm}$. The interactions between curcumin and BTZ were examined via UV-Vis absorption spectra (Fig. 3B). After the addition of BTZ, BTZ/cur showed a distinct absorbance peak at wavelength of $540 \mathrm{~nm}$. This result corresponds with UV absorbance wavelengths of complexes of curcumin and boronic acids such as rosocyanine and rubrocurcumin $[20,21]$. However, the absorbance peak of curcumin and BTZ complexes decreased fast, probably due to poor stability of the BTZ/cur complexes (data not shown).

\section{DPPH assay}

In our previous study, DPBA/cur exhibited higher stability in aqueous solutions and in vivo than free curcumin [14]. However, biological activity of DPBA/cur was not evaluated in terms of antioxidant and antiproliferation effect. To verify influence of DPBA complexation with curcumin on the antioxidant effects of curcumin, a DPPH assay was performed with free curcumin and DPBA/cur comparatively. Ascorbic acid [22], known as a strong antioxidant, was used as a positive control. Figure 4 shows that

Fig. 3 (A) UV-Vis absorption spectra of free curcumin, free DPBA, and DPBA/cur fraction eluted from HPLC at a DPBA/ cur weight ratio of 10. (B) UVVis absorption spectra of BTZ/ cur at various BTZ/cur weight ratios
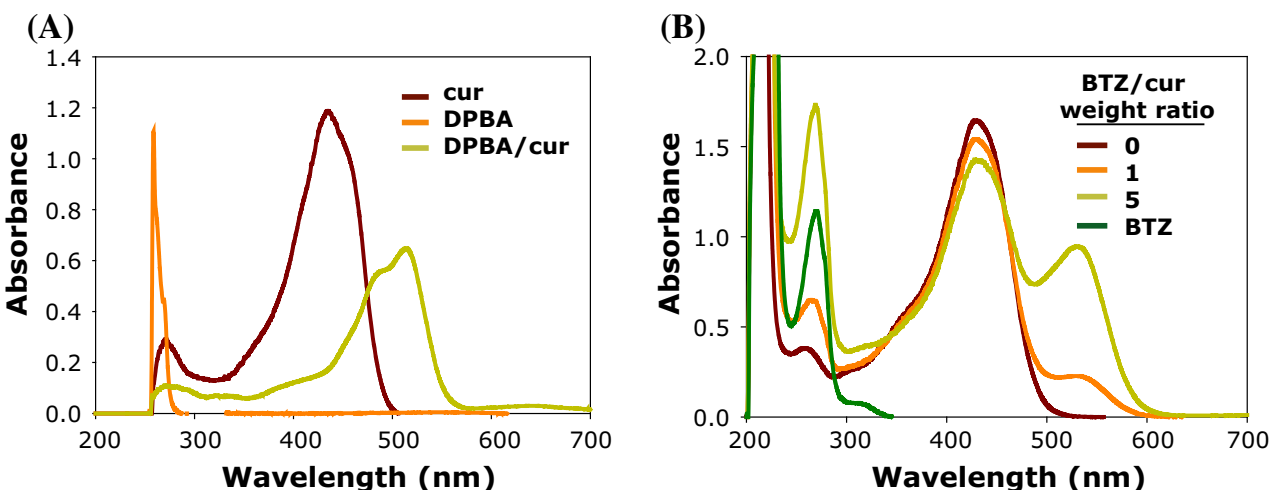

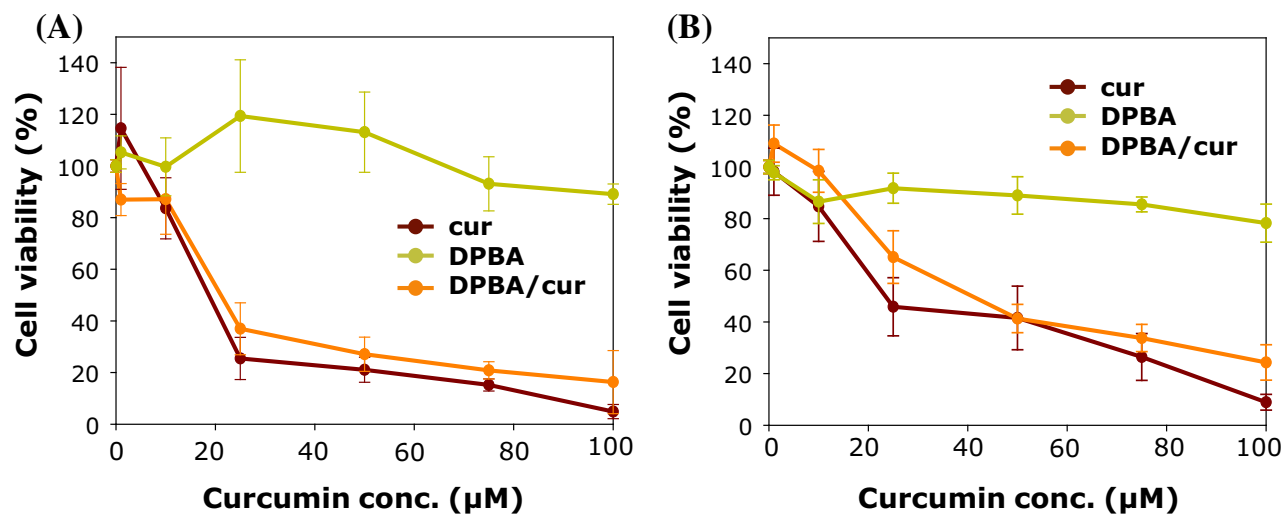

Fig. 5 Cell viability of (A) MCF-7 and (B) A549 cells after treatment with curcumin and DPBA/cur complexes

curcumin and DPBA/cur exhibited similar DPPH inhibition at different curcumin concentrations.

\section{Antiproliferation activity}

Curcumin has been reported to have antiproliferation activity against various tumors via diverse cell signaling $[23,24]$. Previously, many studies reported that the di-keto form of curcumin is involved in antiproliferation activity of curcumin $[25,26]$. The influence of curcumin/DPBA complexation to antiproliferation activity was examined for two kinds of cancer cell lines, MCF-7 and A549. Figure 5 shows that the $\mathrm{IC}_{50}$ of free curcumin were $19.5 \pm 0.6$ and $32.3 \pm 16.5 \mu \mathrm{M}$ for MCF-7 and A549 cells, respectively, which was consistent with previous studies $[27,28]$. IC $_{50}$ values of DPBA/cur at curcumin with a DPBA weight ratio of 1:0.08 were $24.2 \pm 3.9$ and $45.0 \pm 11.0 \mu \mathrm{M}$ for MCF-7 and A549 cells, respectively. There was no significant difference in the $\mathrm{IC}_{50}$ values of free curcumin and DPBA/ cur for both types of cells. Taken together, DPBA complexation has a negligible effect on the antioxidant and antiproliferation effects of curcumin. Considering that DPBA complexation improved the bio-availability of curcumin in vivo in our previous study [14], DPBA complexation could have a great potential to improve the therapeutic effects of curcumin in vivo.

In this study, curcumin was successfully complexed with DPBA and BTZ as determined by HPLC and UV-Vis absorption spectra. The absorbance peak of DPBA/cur and BTZ/cur complexes were redshifted. DPBA complexation has a negligible effect on the antioxidant and anticancer effects of curcumin; this was examined for two types of cancer cells: MCF-7 and A549. Thus, curcumin complexation with boron-based compounds could be a useful strategy for in vivo stability without loss of bioactivity (i.e., antioxidant and antiproliferation effects).
Acknowledgments This work was supported by the Agri-Bio Industry Technology Development Program (316028-3), Korea Institute of Planning and Evaluation for Technology in Food, Agriculture, Forestry, and Fisheries (IPET), Republic of Korea.

\section{References}

1. Kuttan R, Bhanumathy P, Nirmala K, George MC (1985) Potential anticancer activity of turmeric (Curcuma longa). Cancer Lett 29(2):197-202

2. Bar-Sela G, Epelbaum R, Schaffer M (2010) Curcumin as an anticancer agent: review of the gap between basic and clinical applications. Curr Med Chem 17(3):190-197

3. Naksuriya O, Okonogi S, Schiffelers RM, Hennink WE (2014) Curcumin nanoformulations: a review of pharmaceutical properties and preclinical studies and clinical data related to cancer treatment. Biomaterials 35(10):3365-3383

4. Sun DM, Zhuang XY, Grizzle W, Miller D, Zhang HG (2011) A novel nanoparticle drug delivery system: the anti-inflammatory activity of curcumin is enhanced when encapsulated in exosomes. Cancer Res 71

5. Hasan M, Belhaj N, Benachour H, Barberi-Heyob M, Kahn CJF, Jabbari E, Linder M, Arab-Tehrany E (2014) Liposome encapsulation of curcumin: physico-chemical characterizations and effects on MCF7 cancer cell proliferation. Int $\mathrm{J}$ Pharm 461(1-2):519-528

6. Yallapu MM, Jaggi M, Chauhan SC (2012) Curcumin nanoformulations: a future nanomedicine for cancer. Drug Discov Today 17(1-2):71-80

7. Anand $\mathrm{P}$, Thomas SG, Kunnumakkara AB, Sundaram C, Harikumar KB, Sung B, Tharakan ST, Misra K, Priyadarsini IK, Rajasekharan KN, Aggarwal BB (2008) Biological activities of curcumin and its analogues (Congeners) made by man and mother nature. Biochem Pharmacol 76(11):1590-1611

8. Liu YM, Lee K (2009) Modifications of the curcumin method enabling precise and accurate measurement of seawater boron concentration. Mar Chem 115(1-2):110-117

9. Sah RN, Brown PH (1997) Boron determination-a review of analytical methods. Microchem J 56(3):285-304

10. Wimmer MA, Goldbach HE (1999) A miniaturized curcumin method for the determination of boron in solutions and biological samples. J Plant Nutr Soil Sc 162(1):15-18

11. Mair JW Jr, Day HG (1972) Curcumin method for spectrophotometric determination of boron extracted from radiofrequency 
ashed animal tissues using 2-ethyl-1,3-hexanediol. Anal Chem 44(12):2015-2017

12. Baker SJ, Ding CZ, Akama T, Zhang YK, Hernandez V, Xia Y (2009) Therapeutic potential of boron-containing compounds. Future Med Chem 1(7):1275-1288

13. Hunter P (2009) Not boring at all Boron is the new carbon in the quest for novel drug candidates. EMBO Rep 10(2):125

14. Kang YY, Jung H, Yu G, Chong Y, Mok H (2016) Complexation of curcumin with 2-aminoethyl diphenyl borate and implications for spatiotemporal fluorescence monitoring. Int $\mathrm{J}$ Pharm 515(1-2):669-676

15. Medel S, Syrova Z, Kovacik L, Hrdy J, Hornacek M, Jager E, Hruby M, Lund R, Cmarko D, Stepanek P, Raska I, Nyström B (2017) Curcumin-bortezomib loaded polymeric nanoparticles for synergistic cancer therapy. Eur Polym J 93:116-131

16. Aghajanian C, Soignet S, Dizon DS, Pien CS, Adams J, Elliott PJ, Sabbatini P, Miller V, Hensley ML, Pezzulli S, Canales C, Daud A, Spriggs DR (2002) A phase I trial of the novel proteasome inhibitor PS341 in advanced solid tumor malignancies. Clin Cancer Res 8(8):2505-2511

17. Kouroukis TC, Baldassarre FG, Haynes AE, Imrie K, Reece DE, Cheung MC (2014) Bortezomib in multiple myeloma: systematic review and clinical considerations. Curr Oncol 4:e573-e603

18. Park J, Ayyappan V, Bae EK, Lee C, Kim BS, Kim BK, Lee YY, Ahn KS, Yoon SS (2008) Curcumin in combination with bortezomib synergistically induced apoptosis in human multiple myeloma U266 cells. Mol Oncol 2(4):317-326

19. Mujtaba T, Kanwar J, Wan SB, Chan TH, Dou QP (2012) Sensitizing human multiple myeloma cells to the proteasome inhibitor bortezomib by novel curcumin analogs. Int $\mathrm{J}$ Mol Med 29(1):102-106
20. Alberti D, Protti N, Franck M, Stefania R, Bortolussi S, Altieri S, Deagostino A, Aime S, Geninatti Crich S (2017) Theranostic nanoparticles loaded with imaging probes and rubrocurcumin for combined cancer therapy by folate receptor targeting. Chem Med Chem 12(7):502-509

21. Donaldson EM (1981) Spectrophotometric determination of boron in iron and steel with curcumin after separation by 2-ethyl1,3-hexanediol-chloroform extraction. Talanta 28(11):825-831

22. Brand-Williams W, Cuvelier ME, Berset C (1995) Use of a freeradical method to evaluate antioxidant activity. Food Sci Technol-Leb 28(1):25-30

23. Chen A, Xu J, Johnson AC (2006) Curcumin inhibits human colon cancer cell growth by suppressing gene expression of epidermal growth factor receptor through reducing the activity of the transcription factor Egr-1. Oncogene 25(2):278-287

24. Choudhuri T, Pal S, Agwarwal ML, Das T, Sa G (2002) Curcumin induces apoptosis in human breast cancer cells through p53-dependent Bax induction. FEBS Lett 512(1-3):334-340

25. Qiu X, Du YH, Lou B, Zuo YL, Shao WY, Huo YP, Huang JN, Yu YJ, Zhou BH, Du J, Fu H, Bu XZ (2010) Synthesis and identification of new 4-Arylidene curcumin analogues as potential anticancer agents targeting nuclear factor-kappa $\mathrm{b}$ signaling pathway. J Med Chem 53(23):8260-8273

26. Agrawal DK, Mishra PK (2010) Curcumin and its analogues: potential anticancer agents. Med Res Rev 30(5):818-860

27. Simon A, Allais DP, Duroux JL, Basly JP, Durand-Fontanier S, Delage C (1998) Inhibitory effect of curcuminoids on MCF-7 cell proliferation and structure-activity relationships. Cancer Lett 129(1):111-116

28. Radhakrishna Pillai G, Srivastava AS, Hassanein TI, Chauhan DP, Carrier E (2004) Induction of apoptosis in human lung cancer cells by curcumin. Cancer Lett 208(2):163-170 